\title{
Retos y perspectivas de la psicología de la liberación. Consideraciones a finales de siglo ${ }^{1}$
}

\author{
Maritza Montero
}

\section{Resumen}

En este artículo, la autora expone el surgimiento, el desarrollo y la influencia de un modo de construir e interpretar la realidad para transformarla, que si bien se nutre de otras corrientes, pretende generar una respuesta propia, que se inserte en el desarrollo de un paradigma que presenta características específicas en América Latina. Tal desarrollo supone una ontología o concepción del ser; una epistemología, o modo preferente de relación entre el sujeto cognoscente y el objeto conocido; una metodología, es decir, las vías para construir ese conocimiento; una posición ética, en cuanto supone una peculiar relación con el otro y una posición política, puesto que dichas dimensiones afectan a la sociedad y la ciudadanía.

\section{Un poco de historia y algo de definición}

Las ideas sobre liberación son tan viejas como la humanidad. Desgraciadamente, ello es así por- que no menos antigüas son las ideas sobre sumisión y opresión que las hacen necesarias. Pero el contexto en el cual se enmarcarán mis palabras no

1 De la Universidad Central de Venezuela. Conferencia magistral impartida en el Primer Congreso Internacional sobre Psicología de la Liberación, celebrada en México del 16 al 18 de noviembre de 1998. 
se remontará tan atrás, pues sólo deseo señalar las ideas y prácticas que han sentado las bases y que, en algunos casos, han sido precursoras de lo que hoy llamamos "Psicología de la Liberación", a fin de centrarme en ésta.

Asimismo, debo señalar que no me referiré a la teoría marxista, si bien ésta ha sido la corriente fundamental en el planteamiento de formas liberadoras de conciencia y en vías para la emancipación social, al igual que las tendencias críticas de ella derivadas tales como la Escuela Marxista húngara y la Escuela de Francfort, cuyo pensamiento y exponentes marcan las ciencias sociales en este siglo. Tampoco haré un recuento de sus bien conocidos aportes a las teorías de la ideología y de la alienación, como igualmente me abstendré de discutir o analizar los de Agnes Heller o de Jürgen Habermas, últimos vástagos de esos dos núcleos de pensamiento.

Y ello se debe a que circunscribiré mi exposición al surgimiento, desarrollo e influencia de un modo de construir e interpretar la realidad para transformarla, que si bien se nutre de muchos aportes de las corrientes antes mencionadas, va a generar una respuesta propia, insertándose en el desarrollo de un paradigma que presenta características específicas en América Latina. Tal desarrollo supone una ontología o concepción del ser; una episiemología, o modo preferente de relación entre el sujeto cognoscente y el objeto conocido; una melodología, es decir, las vías para construir ese conocimiento; una posición ética, en cuanto supone una peculiar relación con cl otro y una posición política, puesto que las cuatro dimensiones anteriores afectan a la sociedad y la ciudadanía.

La lendencia a la cual deseo referirme es la corriente liberacionista de las ciencias sociales, que emerge en América latina a partir de los años sesenta, si bien su gestación podría ubicarse en los cincuenta y aun antes. Con ella no sólo se construirá un modo de interpretar al mundo, sino que como se ha indicado antes, se está fundando un paradigma. Pero a diferencia del paradigma trunco que nos suelen presentar los textos metodológicos que con ánimo de cambio y espíritu renovador comenzaron a aparecer a mediados de los ochenta en Estados Unidos ( $c f$. Lincoln y Guba, 1985; Guba, 1991), éste va más allá de la ontología, la epistemología y la metodología, incluyendo esos componentes ético y político que son fundamentales para comprender este modelo de producción de conocimiento y para entender de qué trata la liberación.

\section{El concepto de liberación}

Por liberación, en el contexto latinoamericano, entendemos la emancipación de aquellos grupos sociales que sufren opresión y carencia, de aquellas mayorías populares (la palabra "mayoría" y la palabra "populares" se usan en el sentido poblacional, demográfico) marginadas de los medios y modos para satisfacer dignamente las necesidades tanto básicas como complementarias, y para desarrollar sus potencialidades para autodeterminarse. También abarca esa liberación la emancipación de los grupos opresores, respecto de su propia alienación y dependencia de ideas socialmente negativas.

Liberación entonces, no es una cosa, no es un momento, no es un don ni es una dádiva que se extinguen en un acto. Se trata de un movimiento y de una serie de procesos. Freire (1970) lo considera como una conquista y como un proceso que tienen doble origen: en agentes externos cuya función es catalizar la transformación, y en los propios grupos oprimidos, sometidos, que son agentes internos de su propio cambio. Estc es un aspecto fundamental, esencial para la comprensión de esta corriente, que la dislingue de otras que han planteado la necesidad de cambios sociales estructurados desde fuera de la situación por cambiar.

Si hicn, como he dicho, sus iniciadores luvieron, como la han tenido la mayoría de los investigadores sociales del siglo XX, la influencia de las ideas de Marx y Engels y de sus seguidores, aquí hay, ya de inicio, un avance respecto a las Icorías, tales como la teoría de la ideología o la de la alienación. Así, el agente externo no es, como en el caso del "intelectual orgánico" del cual hablaba Gramsci (193I/1984) alguien que va a iluminar a las masas, hasta ese momento a oscuras. Tampoco esas masas están compuestas de entes carenciados que no poseen ninguna fuerza, ninguna capacidad de acción o de reacción. No son un rehaño por conducir ni están sordos, ciegos, mudos o paralíticos. La idea de la liberación que surge en América Latina propone una alianza estratégica entre dos tipos de actores sociales igualmente activos y supondrá una reconceplualización de la teoría del poder, como veremos en la década de los ochenta. 


\section{Orígenes}

Las ideas iniciales para esta concepción se pueden situar en dos escuelas dentro de las ciencias sociales, en ninguna de las cuales aparece inicialmente presente la psicología, aun cuando hay muchas ideas psicológicas en ellas. Me refiero a la Educación Popular freiriana y a la Sociología Crítica o Militante de Fals Borda. Ambas se inician en prácticas transformadoras y tienen el cambio social como meta, pero centran ese cambio no en la sociedad en abstracto, sino en individuos y grupos concretos.

Y en ambas corrientes, que tienen muchos aspectos coincidentes, sus partidarios a la vez que practicantes se definen como agentes de cambio social, no como expertos dueños del único saber en juego. Esto supone redefinir el rol de los profesionales, sean educadores, sociólogos, trabajadores sociales, psicólogos, trabajadores de la salud, sacerdotes o meros interesados. La redefinición se da en el sentido de que dejan de ser considerados como la autoridad única rectora del proceso de cambio, para ser catalizadores o facilitadores, que si bien poseen habilidades y conocimientos específicos, deben igualmente olvidar la denominación de "sujeto" con la cual tradicionalmente se definía a aquellas personas a las cuales incluíamos en las investigaciones de carácter extractivo, que dominaban el pensamicnto cientílico hasta hace pocos años. La liberación entonces comienza aquí, en ese acto de reconocimiento de la libertad del otro, que deja de ser un sujeto "sujetado" para ocupar un sitio de igualdad, como actor social fundamental propietario de habilidades y conocimientos específicos de una índole diferente. Esto lleva al reconocimiento de que, junto a un saber científico, técnico, especializado, existen otros saberes, propios de los grupos con los cuales se trabaja. Por lo tanto, una y otra corricnte incorporan la valoración del saber popular, el respeto por él y la necesidad imprescindible de tomarlo en cuenta.

En el Cuadro 1 se resumen los aportes de estas dos corrientes, y se resaltan en cursivas aquellos aspectos coincidentes en ambas, que luego encontraremos en el desarrollo posterior de la psicología de la libcración. Esos aportes pueden resumirse en los aspectos siguientes, de los cuales ya se han mencionado tres: generación de una praxis liberadora; centrada en la transformación social; acción transformadora sobre la realidad; con redefinición del rol de los investigadores e interventores sociales; y definición de las personas y grupos interesados como actores sociales; relación dialógica entre agentes externos y agentes internos del cambio social; valoración del saber popular; desideologización; concientización; autocontrol por parte de las personas y grupos interesados; compromiso; uso de formas participativas de investigación-acción; recuperación crítica de la historia.

En el caso del concepto de concientización, introducido por Paulo Freire $(1964 ; 1970)$, su definición marca una diferencia con el pensamiento europeo que hasta el momento dominaba la visión liberadora, y en el cual el propio Freire se nutre, pero al cual no repite de manera acrítica. Así, por concientización no se entiende desarrollar, crear o generar una forma de conciencia allí donde no la había, sino el movimiento de la conciencia que la lleva a transformarse de ingenua a crítica, en el proceso de conocer las relaciones causales y circunstanciales que originan determinados fenómenos sociales. Movilización que se produce a través del diálogo y de la problematización de las circunstancias colidianas. Y como ya se mencionó en otra parte (Montero, 1991), la concientización no es un acto único, imperecedero, sino un proceso continuo, una construcción de cada día, en constante tensión con la presión conservadora que se construye, deconstruye en la práctica, y también se puede destruir en ella.

Sobre estas bases, la psicología comienza, a mediados de los años setenta, a intentar redefinir su praxis social, y trata de responder así a las críticas y acusaciones de ser socialmente irrelevante $\mathrm{c}$ insensible. Tal examen de conciencia, así como el dolor de tales pecados y su correspondiente propósito de enmienda, se traducirán en el surgimiento de una corriente de construcción y transformación crítica, marcada por ideas liberadoras en el campo de la psicología comunitaria, cuyo desarrollo inicialmente concretado a formas de intervención, ha 
Cuadro

Influencias teórico-prácticas

\section{Propuesta liberadora de Paulo Freire}

1970
Propuesta liberadora de

Orlando Fals Borda

1959-1978-1981-1985

\section{De la opresión a}

\section{Opresión}

Prescripción

Comportamiento prescrito

conformismo

Alienación

Miedo a la libertad

Adaptación.

Conciencia servil

Gregarización

Autodesvalorización

Racionalización

"Nadie libera a nadie, ni nadie se libera solo".

\section{la liberación}

Pedagogía del oprimido

Praxis liberadora. Relación dialógica con agentes externos.

Conciencia de la opresión

\section{Concientización}

Inserción crítica de oprimidos en la realidad opresora.

Problematización, desnaturalización.

Convivencia

Acción sobre la realidad

Esperanza

Reflexión

Valoración del saber popular.

Liberación (conquista y proceso).

Compromiso.

Cambio en el rol de los agentes externos.
Acción comunal como nueva praxis para transformar la realidad.

Uso de investigación-acción, luego participativa al incorporar a miembros de las comunidades.

Catálisis social.

Autonomía del grupo.

Prioridades determinadas por el grupo.

Realizaciones: acciones materiales, desarro-

llo de la conciencia.

Estímulos (materiales e inmateriales).

Cambios en la concepción del mundo.

Cambios en la personalidad social (sic).

- Rechazo a la sumisión.

Autocontrol.

- Dignidad.

Aprender a dirigir.

- Disminución de autoviolencia.

- Pérdida del temor a innovaciones. Apertura al cambio.

- Análisis de riesgos y beneficios.

- Conciencia comunal.

Concientización.

Superación de la pasividad.

Cambio social: ambiente y seres humanos.

Cambio en rol de agentes externos.

Compromiso

Recuperación crítica de la historia.

Devolución sistemática del conocimiento producido. 
pasado a la construcción teórica y a la disquisición epistermológica, así como a ser objeto de aplicación y estudio en otros ámbitos. $Y$, por otra parte, ha producido una reconducción de la psicología política, en el sentido de pasar a ocuparse de los problemas propios de estas sociedades, precisando en tal sentido deslastrarse de teorías, conceptos y métodos que en lugar de contribuir al conocimiento de los fenómenos psicosociales que afectan el campo político y son afectados por él, en nuestros países más bien contribuían a ocultar su sentido, sus causas y sus consecuencias.

Una tercera influencia, no menos importante, es la de la teología de la liberación, una forma de pensamiento desarrollada en América Latina e igualmente marcada por el paradigma antes mencionado, en el cual asimismo ha dejado sus señales. La teología plantea una concepción renovadora de la Iglesia católica, pues busca el conocimiento de Dios a través de conocer y mejorar la existencia de los seres humanos, por lo cual trata de responder a los problemas sociales de este continente. La teología de la liberación asume con extraordinaria fuerza la idea de concentrar los esfuerzos en la atención a las mayorías oprimidas, en los desposeídos, pues considera que en la voz de ese pueblo está la voz de Dios. Esta posición nuevamente lleva a señalar la necesidad de establecer un compromiso social con los necesitados y con una transformación social orientada hacia la justicia, por lo cual es fundamental tomar en cuenta el contexto social de la acción. Se otorga así un carácter político a la práctica teológica, a la vez que élico, y se anuncia (Boff, 1993) que la praxis es cl asiento de la verdad. La praxis como criterio de verdad va a suponer, a su vez, una relación dialéclica entre ella y la teoría, que deberá construir y revisar sus postulados en función de la acción reflexiva.

Igualmente es necesario mencionar las propuestas que hacía la psicología comunitaria, que se había iniciado en muchos países latinoamericanos a mediados de la década de los setenta, en la cual igualmente es posible encontrar la marca del mismo paradigma, y que ya, desde finales de dicha década e inicios de los ochenta, publicaba resultados de formas de intervención psicosociales hechas con una motivación transformadora de la realidad, centradas en los actores sociales y llevadas a cabo con ellos y no para ellos, situando en la comunidad el centro del poder y del control, con redefinición del rol de los psicólogos/as y con una definida intención emancipadora.

En este ámbito, marcado por una renovación de la acción y de la ciencias sociales, es donde sonará por vez primera el nombre de psicología de la liberación.

4. Una psicología de la liberación liberadora: el pionero, Ignacio Martín-Baró

En 1986, Ignacio Martín-Baró, psicólogo salvadoreño nacido en España, pero cuya acción y devoción lo ligaron a esa tierra que regó con su sangre, publicó, en el Boletín de Psicología de la Universidad Centroamericana "José Simeón Cañas", un artículo que tituló "Hacia una psicología de la liberación". Allí, después de hacer un análisis de la situación político social de los países latinoamericanos, y del rol que debería cumplir en ellos la psicología, proponía tres bases sobre las cuales debería erigirse esa psicología (1986, pp. 22-23): (a) "Propiciar una forma de buscar la verdad desde las masas populares", como lo propone la teología de la liberación. (b) Crear una nueva praxis psicológica para transformar a las personas y las sociedades, reconociendo sus "potencialidades negadas" (1986, pp. 22-23). (c) Descentrar la atención de su status científico, para dedicarse a los problemas urgentes de las mayorías oprimidas en América Latina.

En estas tres condiciones es posible ver la influencia teológica liberacionista y las influencias freiriana y falsbordiana que, por otra parte, están explicitadas tanto en ese texto cuanto en otros que lo siguieron. Una conferencia dictada en 1987 en la Universidad de Costa Rica, sobre cuyo conteni- 
do volvería a hablar en 1989 en la Universidad de Guadalajara, "Retos y perspectivas de la Psicología en América Latina" (1987/1989/1991), perfila un poco más estas ideas, a la vez que profundiza más en su condición político social. El cobarde asesinato de Ignacio, a manos de soldados del ejército salvadoreño en noviembre de ese mismo año, nos privó de ulteriores desarrollos y de la clara visión de un psicólogo comprometido profunda y totalmente con un pueblo, con una sociedad y con una concepción de hacer ciencia desde una psicología que entendía como social y política, y con una labor liberadora hecha desde las personas y grupos necesitados de ella. En sus propias palabras: "la liberación debe hacerse desde los oprimidos, no para los oprimidos" (1989).

Esta es una idea fundamental en la psicología que proponía y que se evidencia en toda su obra: Hacer una práctica marcada por el lugar y por su gente. Por eso titula su libro sobre Psicología Social: Psicología social desde Centroamérica, algo que Blanco (1993) ha sabido señalar al destacar el carácter histórico que Martín-Baró defendía para la disciplina.

Si bien es poca la producción psicosocial, que sobre esta forma de hacer psicología dejó MartínBaró, es posible extraer de ella ciertas ideas orientadoras que hablan de su riqueza y de la evidente rellexión, obviamente proveniente de una praxis de la cual se desprende. Esas ideas son las siguientes:

1. Tin cuanto a la incorporación de las masas populares y de su saber, Martín-Baró señalaba la necesidad de: $(a)$ potenciar las virtudes populares. (b) El estudio de lo que llamaba los "grupos con historia" (Martín-Baró, 1988), es decir, organizaciones populares que debían ser estudiadas en su realidad, en su carácter histórico y en sus aspectos cualitativos, en lunción de tres dimensiones esenciales: su identidad, su poder y su actividad (1988, pp. 6-7). (c) El esludio sistemático de las formas de conciencia popular, respecto de la cual proponía la exploración de nuevas lormas de expresión. (d) Desideologizar el sentido común y la experiencia cotidiana, consciente como estaba de que lambién en cllos hallan asiento el error y la conlusión, al lado de la crílica y la innovación.

2. En cuanto a la creación de una praxis psicológica translormadora liberadora: (a) Desarrollar formas de control por parte de los grupos oprimidos. Lo que Fals Borda ha llamado "autonomía del grupo" (1959). (b) La desalienación social y personal. (c) Superar el fatalismo existencial, tema sobre el cual publicó en 1987 un agudo análisis. (d) Estudiar el problema del poder.

3. En cuanto al rol de la psicología, para ella señala tres tareas: $(a)$ Dedicarse a los problemas urgentes que plantean las sociedades latinoamericanas. Es decir, separarse de los problemas derivados de los libros y revistas científi$\cos$ y atacar aquellos que sufre la gente. Recordemos que para los años ochenta, todavía había quien sostenía que la tarea de los psicólogos en nuestros países debía ser replicar los estudios realizados en los centros académicos de Estados Unidos y de Europa, a fin de demostrar si las hipótesis y teorías creados en ellos eran o no aplicables en nuestro medio (Rodríguez, 1972). Algo que podríamos denominar como la propuesta de la zanahoria delante del asno. (b) Fomentar la recuperación de la memoria histórica en las mayorías oprimidas, lo cual contribuirá a la desalienación y desideologización. (c) Los psicólogos deben comprometerse con la transformación social que libere a los oprimidos de las condiciones que les oprimen.

\section{Perspectivas de la psicología de la liberación}

Después de la trágica mucrte de Ignacio Marlín-Baró, si bien muchas acciones que responden a las características liberadoras propuestas, tanto por la tradición de las ciencias sociales latinoamericanas como por sus propuestas iniciales, se han realizado y realizan en muchos países del continente, e incluso existen algunas instituciones que incorporan a su nombre la palabra liberación (por ejemplo, el Núcleo Internacional de Estudos c FormaVao cm Psicología Comunitária da LibertaVao, en Brasil), es muy poco, por no decir casi nada, lo que se ha publicado en esos mismos países. En 1991, la autora publicó una propuesta sociopsicológica para una psicología de la liberación, que correspondía a una conferencia dictada en la Universidad de Hamburgo en 1990. Ignacio Dobles, en Costa Rica, igualmente ha Irabajado sobre el tema. Pero hasta donde puedo saber, no ha habido mucho más. Sin embargo, la idea de la liberación en psicología, con o sin la influencia de Marlín- 
Baró, se puede sentir tanto en la práctica, sobre todo comunitaria y política (por ejemplo en la defensa y estudio de movimientos políticos originados en minorías) donde reside su actual fuerza, como también en producciones teóricas paralelas.

En tal sentido mencionaré dos, de las cuales existe la huella perenne que constituyen las publicaciones. La primera es la "Psicología de la opresión", propuesta por Isaac Prilleltensky en 1993; la segunda, los prolegómenos a una psicología de la liberación, de Thomas Theo, aparecida hace apenas un par de meses.

La psicología de la opresión. Si bien, como ya se deduce de su propio nombre, esta propuesta está hecha desde la situación negativa que sufren los oprimidos, más que desde la posición positiva de su liberación, la propuesta de Prilleltensky -investigador canadiense nacido en Argentina, donde vivió hasta los 14 años; con estudios superiores en Israel de donde emigró al Canadá- constituye una psicología de liberación, que además puede decirse que se ubica dentro de la misma línea que se ha venido desarrollando en América Latina. Para este investigador, los clementos fundamentales de este modo de hacer psicología deben ser (ver Cuadro 2):

(1) El cstudio del poder como clemento clave, tanto del mantenimiento y preservación del orden social como de su Iransformación y cambio.

(2) La promoción de la cactividad de los grupos oprimidos, incorporándolos a las acciones transformadoras, las cuales no pueden ser llevadas a cabo sin ellos. Actividad que debe ser considerada como una práctica político. (a) Esa actividad debe centrarse en prácticas liberadoras. (b) La práclica psicológica debe dedicarse al fortalecimiento o potenciación (empowerment) de los grupos deslavorecidos, a lïn de que puedan reclamar sus derechos $y$ defender sus intereses. (c) Debe generarse un compromiso ético con los oprimidos. (d) Revitalizar las prácticas comunitarias. (e) Concientización, a lin de comprender cómo se generan las formas de consentimiento tácito a las práclicas sociales opresoras, y el conformismo que lleva a la aceptación de las hegemonias. $(f)$ Denunciar la injusticia social. (g) Fomentar formas de resistencia organizada y de lucha contra los modelos dominantes y opresores de ciencia, de salud mental, de orientación sexual, de relaciones de género, de lenguaje opresor, etc. $(h)$ Promover el control de su situación de vida en los grupos oprimidos.

(3) Enfasis en los aspectos éticos y morales. Como se puede ver, ésta es una posición altamente coincidente con los postulados a los cuales nos hemos venido refiriendo hasta ahora. Poder, fortalecimiento, cambio social, compromiso ético, concientización y acción política y las relaciones que entre ellos se postula, son suficientes elementos para identificar esta teoría como una expresión de la psicología de liberación.

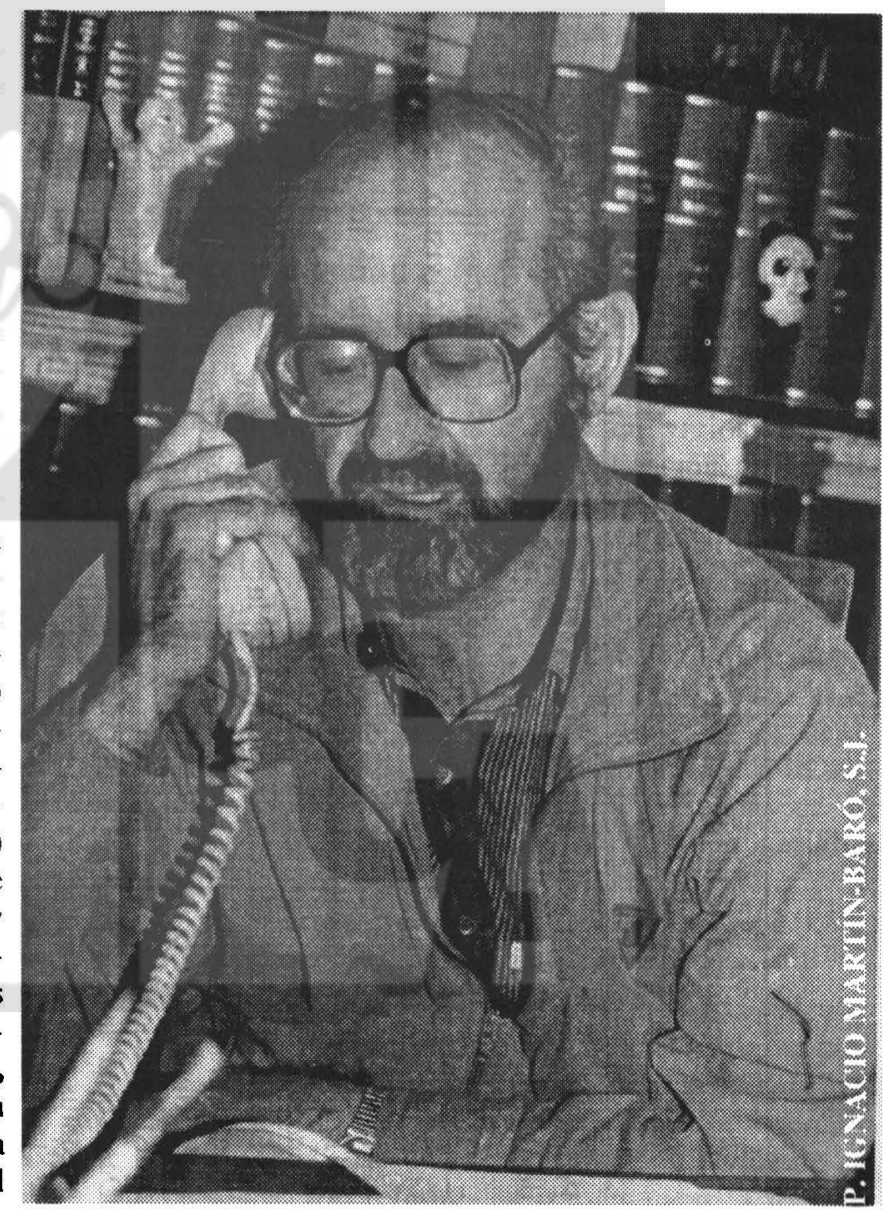


Cuadro 2

Psicologías de la liberación

\section{Psicología de la liberación \\ Ignacio Martín-Baró}

1986-1989

\section{Psicología de la opresión \\ Isaac Prilleltensky}

\section{Psicología de la liberación \\ Thomas Theo}

1998
Propiciar una forma de buscar la verdad desde las masas populares.

- Potenciar las virtudes populares.

- Análisis de las organizaciones populares como instrumento de liberación histórica.

- Estudio sistemático de las formas de conciencia popular.

- Desideologizar el sentido común y la experiencia cotidiana.

Crear una nueva praxis psicológica para la transformación social e individual.

Descentrar la atención de la psicología, de su status científico.

- Dedicarse a los problemas urgentes de América Latina.

- Recuperar la memoria histórica.

Elementos para construir esa psicología:

- Control.

- Desalienación social y personal.

- Superar el fatalismo existencial.

- Compromiso con la transformación y con los oprimidos.

- Exploración de nuevas formas de conciencia.

- Tomar en cuenta el problema de poder.

La liberación debe hacerse "desde" los oprimidos, no "para" los oprimidos".
El poder como un elemento clave en la preservación y cambio del orden social.

Promover prácticas liberadoras.

Promover el activismo de los grupos oprimidos.

Fortalecimiento de los desfavorecidos para afirmar sus derechos e intereses, y medio para lograr un compromiso ético con los oprimidos.

Revitalizar prácticas comunitarias adormecidas o relegadas a un segundo lugar.

Promover la resistencia organizada.

Expandir las oportunidades de desarrollo a través de la concientización y de la acción política.

La concientización trata de comprender cómo se da un consentimiento tácito al actual sistema social, cómo se produce el conformismo con formas de hegemonía.

Denuncia de la injusticia social.

Lucha contra modelos dominantes y opresores de ciencia, salud mental, género, orientación sexual.

Lucha contra el lenguaje de la opresión.

Entender que lo individual también es político. Nueva agenda de control.

Lo social y lo político son inherentes a la psicología.

Enfasis en los aspectos éticos y morales.
Liberación en función de las posibilidades individuales, aun cuando pueda haber cooperación del otro.

Parte de una teoría crítica de la subjetividad.

Sigue la tradición europea:

- Marxista (Marx, Engels, Holzkamp).

- Neomarxista (Habermas).

- Postmarxista (Foucault).

Enfoque individualista.

Su ámbito son las áreas en que el sujeto puede estar activo.

Imprescindible tomar en cuenta las relaciones de poder.

La teoría como un instrumento práctico. 
Los prolegómenos para una psicología de la liberación. Bajo este título, Thomas Theo, profesor asistente en la Universidad de York, en Canadá, con formación doctoral en la Universidad de Viena, se propone sentar las bases para una psicología de la liberación inspirada en la tradición europea que, según indica en su obra, es aquella que deriva del marxismo, traducido en términos psicológicos por Klaus Holzkamp; del neo-marxismo, encarnado por la teoría de Jürgen Habermas, y del post-marxismo, expresado en la obra de Michel Foucault.

El centro de su propuesta reside en el concepto de poder, el cual necesita ser estudiado para poder comprender la necesidad de la liberación. Esto debe hacerse analizando los tres paradigmas que derivan de las corrientes antes mencionadas. En el primer caso, sería el paradigma del trabajo como relación en la cual se puede manifestar el poder. En el segundo caso, sería el paradigma de la interacción y, en el tercero, el paradigma que denomina estélico, referido a la valoración del poder en la construcción del sí mismo.

La liberación es definida como el lado psicológico o subjetivo de un proceso dialéctico. El otro lado sería el contexto. $Y$ en sus palabras: "una psicología de la liberación toma en cuenta el lado del sujeto" (p. 539). Tanto, que la liberación es presentada como una decisión personal y el fortalecimiento es visto "primordialmente como una competencia individual" (1998, p. 537). Así, al ser confrontado el sujeto por una siluación de opresión, decide si quiere luchar contra ella, para lo cual debe analizar el contexto relacionando sus acciones con algunos de los paradigmas señalados: trabajo, interacción o la autovaloración en la intrasubjetividad, lo cual orientará su reflexión y las decisiones y acciones que realice. En este sentido, la teoría es considerada como "un instrumento práctico". Pero un instrumento destinado a quienes van a ser sujetos activos de la liberación de otros implícitamente pasivos.

En este caso estamos ante una propuesta en la cual la liberación, así como su carácter político y ético, tal como la hemos venido considerando, poco o nada tienen que ver. Liberación para Theo es un contenido al cual el sujeto dirige o no su atención y acción. Un objeto de atención racional. $\mathrm{Y}$ es así como se muestra la obra de Theo. Como un objeto de interés sobre el cual hace un estudio no comprometido, desde una posición que nada tiene que ver ni con la opresión ni con la liberación.

Ignorando las prácticas desarrolladas, si no por la psicología de la liberación existente hecha en América Latina, tan poco abundante en publicaciones, si por los aportes de Freire y de Fals Borda, ampliamente traducidos y conocidos, Theo dice que la psicología de la liberación de la cual se ocupa refleja la "tradición de pensamiento europeo continental crítico" (1998, p. 529). Una revisión un poco más amplia le habría revelado cómo el marxismo, el neomarxismo habermasiano y el postmarxismo foucaultiano, al ser analizados críticamente pueden dar lugar a una teoría de la liberación autóctona, con una práctica enriquecedora.

Esto nos muestra que estamos en una situación en la cual poseemos una perspectiva clara, con raíces multidisciplinarias, que se manifiesta en una práctica rica, fuerte y amplia, expresada en varios campos de aplicación de la psicología: la política, las comunidades, donde halló terreno propicio. $Y$ que debería hacerse sentir más en los campos de la salud, la educación, el trabajo, entre otros. Pero, al mismo tiempo, ya se anuncian los desarrollos que pueden definir a la liberación a la manera tradicional, despojando al concepto de su esencia misma, convirtiéndolo en una variable motivacional más.

\section{Los retos de la psicología de la liberación}

Dos tipos de retos se le presentan a la psicología de la liberación. Retos que derivan de su pro pia construcción en respuesta a un problema humano y social, y retos que derivan de su inserción en lo social y, a la vez, en el mundo de vida de las personas. Me referiré, en primer lugar, a los pri- 
meros, que llamaré retos estructurales o retos internos.

(1) Un primer reto es la necesidad de aplicar el principio de la devolución sistemática del conocimiento producido (Fals Borda, 1985) y el de la acción-reflexión-acción (Freire, 1964; 1970), no sólo a los grupos y comunidades con los cuales se ha aplicado, sino también a otra comunidad con la cual igualmente tenemos un compromiso, no menos político y ético: la comunidad científica. Si no sistematizamos ese conocimiento y lo damos a conocer, no sólo estaremos privando a otros investigadores e interventores sociales de la posibilidad de enriquecerse con lo que presentamos, sino también de evitar los errores que podamos haber cometido. Y, a la vez, nos privaríamos de la crítica y de su efecto depurador y fortalecedor. No podemos criticar a un autor por desconocer una posición, si ella no ha sido dada a conocer. Por otra parte, las teorías que producimos tienen ciertamente un efecto práctico, pueden enriquecer a través de las reflexiones que suscitan nuestra práctica.

(2) Un segundo reto es dirigir la reflexión crítica hacia el propio concepto de psicología de la liberación. ¿Se trata de una nueva rama de la psicología? ¿De un campo de aplicación psicológica? ¿De una orientación filosófico-psicológica? Esto es algo que la misma reflexión traducida en obra podría aclarar. Como van las cosas, parece que estamos ante una manera de hacer psicología. Esto significa que podría haber una psicología clínica liberadora, una psicología política liberadora, y así sucesivamente. Que no se trata de una subdisciplina con un objcto, un método, un lenguaje, un marco conceptual, propios y distintos. En tal sentido, la psicología de la liberación es más un paradigma y, como tal, modela, dirige, muestra una vía y señala modos preferentes de hacer. Como paradigma podría marcar entonces a cualquier quehacer psicológico y señalar pautas.

(3) Otro reto, y es el trabajo de Theo el que da la señal de advertencia, es evitar el privilegiar la estética sobre la ética, de tal manera que una decisión "bondadosa", con una tendencia "afiliativa" o "caritativa", o simplemente "bien intencionada", substituyan, como a veces ocurre, una psicología de la liberación por un activismo de la liberación. En el segundo no hay reflexión sistemática, no hay crítica ni hay producción de conocimiento científico generalizable. Posiciones de ese tipo abundan y no configuran una forma de ciencia.

(4) Lo anterior conlleva a un nuevo reto: la necesidad de adoptar, mantener y desarrollar una perspectiva crítica que ayude a evitar las ritualizaciones alienantes, la transformación del calificativo "liberador" y del apelativo "de la liberación", en meras etiquetas destinadas a cubrir prácticas repetitivas en las cuales, bajo la denominación, se vuelvan a guarecer el abuso, la opresión y la exclusión del otro.

(5) Catalizar el desarrollo del carácter ciudadano de los agentes internos con los cuales trabajamos, a fin de que su conciencia y su acción sean una fuente de conocimiento y de crítica. Esto supone profundizar en la tarea de definición y auto-afirmación de los actores sociales, para lo cual los/las psicólogos/as debemos prestar los recursos de nuestro saber especializado, incorporando, al mismo tiempo, el saber y la historia de esos actores sociales.

(6) $\mathrm{Y}$ para poder atender a ese reto, es necesario simultáneamente enfrentar y atender a otro: la necesidad para los psicólogos/as de desarrollar y mantener una actitud de apertura al conocimiento, cualquiera que sea su origen; de curiosidad en el sentido de estar alerta ante las contradicciones, los vacíos, los silencios silentes de la ignorancia y los silencios rumorosos que dicen ocultando. Las palabras vacías y aquéllas cargadas de significado. Es necesario entender que el conocimiento se construye en el diálogo $y$, por lo tanto, es fundamental aprender el difícil arte de dialogar, de escuchar al otro.

Los retos planteados por la inserción social de la psicología de la liberación, que llamaré retos funcionales, y si la simetría nos apremia, retos externos, derivan de los aspectos siguientes:

(1) En primer lugar de la necesidad de conceptualizar y definir al poder. La concepción, el ejercicio y el enfrentamiento al poder son fundamentales para el ejercicio de esta psicología. Algo nada fácil, puesto que tratar con el poder supone no sólo participar e involucrarse en situaciones de riesgo, sino además, como lo proponen Serrano-García y López (1991), hacer una reconceptualización del mismo, oponiendo a su concepción asimétrica una perspectiva negocia- 
dora a partir de recursos propios de los grupos en desventaja, usualmente no sólo no considerados ni mencionados, sino además negados incluso por quienes los poseen. Esto supone, por una parte, despojarse de las concepciones asimétricas que sólo ven el poder en un polo de la sociedad y lo consideran implícitamente omnipotente, con lo cual debilitan a los oprimidos. Y, por otra parte, supone desarrollar las destrezas necesarias para saber captar los recursos a partir de los cuales negociar, junto con los actores sociales involucrados, las soluciones liberadoras.

(2) Una corriente parece estar recorriendo no sólo a América Latina, sino también a otras zonas del globo: la necesidad de redefinir la democracia. El modelo representativo de democracia parece estar agotado y tanto politólogos como científicos sociales en general y políticos en particular, con mayor o menor regocijo o preocupación, según el caso, anuncian o denuncian el fenómeno. Es más, a la tradicional forma representativa ha comenzado a oponerse otra, la democracia participariva. Esta es presentada como una forma, aún poco clara en su implementación, de dar voz a los oprimidos, de abrir las puerlas a los excluidos, de permitir a las grandes mayorías silenciosas y a las grandes minorías silenciadas expresar sus nccesidades y acceder a los recursos necesarios para satisfacerlas en igualdad de circunstancias. Una psicología de la liberación tiene necesariamente que tomar en cuenta este fenómeno y participar en la generación de modos de articular esa participación democrática. En particular, si se toma en cuenta la dificultad inherente a la contradicción entre participación y crecimicnto demográfico, que presenta un obstáculo muchas veces considerado como insalvable. Pero porque existen tales dificultades existc la ciencia y una psicología que prelenda liberar no puede ir a la zaga, ni contentarse con la sola reproducción de los modos usuales de enlrentar los problemas; debe ser creativa, y ante cambios de este tipo debe generar y catalizar conocimienlos necesarios para dar signo positivo al cambio.

(3) Lo anterior supone una confrontación más especílica entre Estado e individuos, algo a lo cual en América Latina, la psicología social comunitaria junto con la psicología política están intentando responder. Tales respuestas deben incorporar el enfoque liberador, so riesgo de convertirse en vías de adaptación o de modulación de esa relación que, en nuestros países, puede ser en particular difícil, cuando no francamente opresora. Por lo tanto, un reto para la psicología de la liberación, en este campo, es el de generar los modos de desarrollar y fortalecer a la sociedad civil, construyendo una ciudadanía consciente de sus derechos y cumplidora de sus deberes. $\mathrm{Al}$ hacerlo, se facilitará el logro de una democracia participativa eficiente.

(4) Las tendencias sociales de este fin de siglo no pueden ser más complejas. Al individualismo, para el cual la concepción positivista preparó al mundo occidental, y que ha reforzado la implantación del neoliberalismo, se enfrenta un creciente comunitarismo que ya tuvo una fracasada incursión utópica e idealista entre finales de los años sesenta e inicios de los setenta, pero que ahora, y en particular como se está planteando en regiones periféricas, tales como nuestra América, vuelve a emerger no sólo con renovada fuerza, sino además redefinido en función de problemas sociales reales y apoyado en ideas, tales como las de la liberación. Pero la coexistencia de neoliberalismo y de comunitarismo, de liberación y de libertad para consumir; de calidad total y de estructura jerárquica e imperios empresariales, genera no sólo la confusión sino las contradicciones que hacen urgente el desarrollo de una conciencia crí-

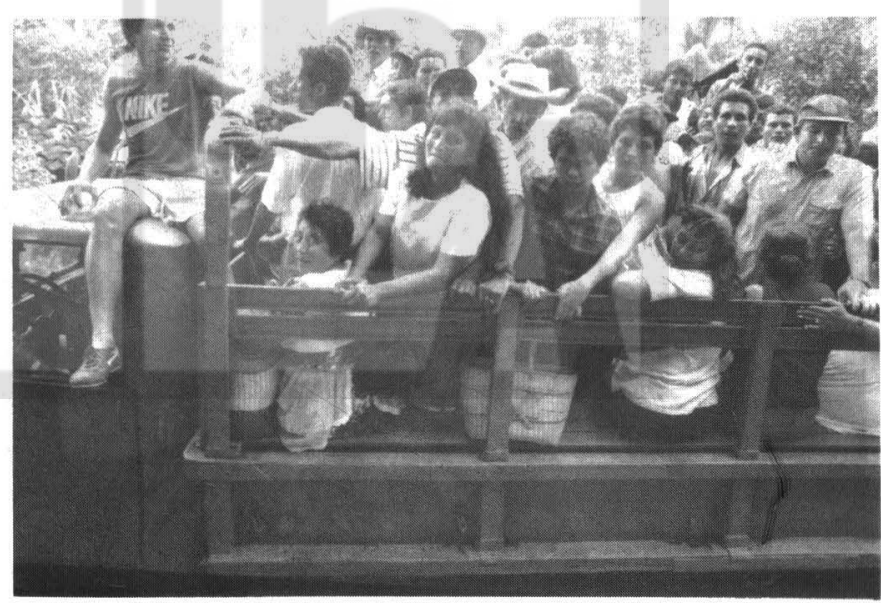


tica, que permita a individuos y grupos sobrevivir a las presiones simultáneas y apremiantes de signo contrario. Nuevamente, ante los dilemas de nuestro tiempo, la psicología de la liberación tiene el reto de presentar modos viables que permitan a los oprimidos desarrollar su capacidad crítica e implementar respuestas liberadoras.

\section{Conclusión que no pone fin a lo que apenas comienza}

Si algo bueno tiene el vivir en momentos de cambio (cambio social, cambio político, cambio paradigmático, cambios científicos) es la indefinición de las circunstancias de vida, el carácter borroso que asume el mundo de vida. Esas mismas condiciones son las que hacen terribles los momentos de cambio. Ya lo decía Rilke: Todo ángel es terrible. Si estamos girando arrastrados por la tromba de los cambios, nos será muy difícil prever cómo y cuándo acabará todo el asunto. Pero sí es posible detectar algunos de los elementos que responden a las corrientes en movimiento.

Tal ocurre con esta mezcla de procesos psicológicos y fenómenos sociales que han suscitado la propuesta de una psicología de la liberación. Y ya en América Latina podemos vislumbrar el surgimiento de modos de acción y de respuestas retlexivas, de corrientes de pensamiento que señalan vías en un mismo sentido, y que suponen cambios fundamentales y necesarios para introducir una nueva concepción del mundo y del ser en el mundo. Me refiero no sólo a la misma idea de la liberación construida desde el oprimido mismo, a la vez que desde fuera de él, sino a la presencia previa de una teología y de una filosofía de la libera-

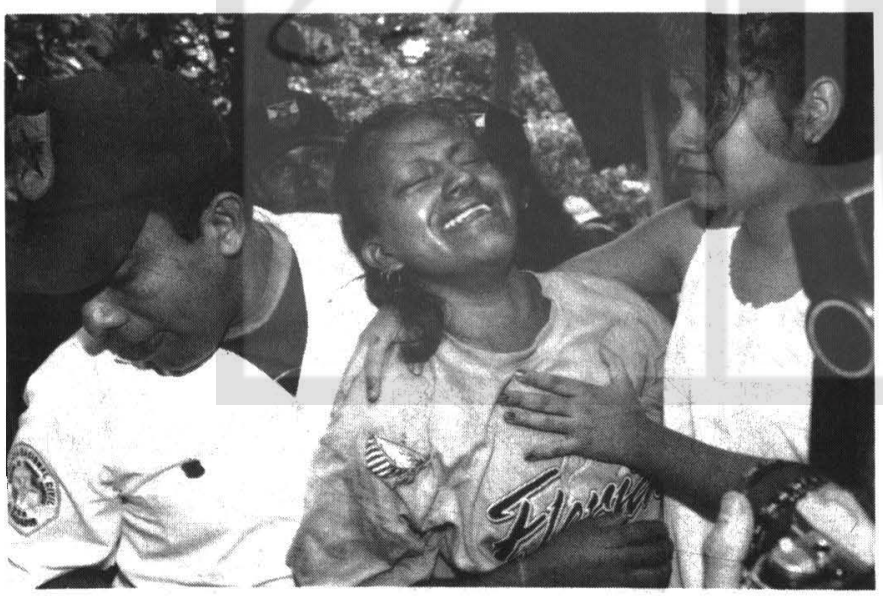

ción. Y unidas a ellas tantas investigaciones aparentemente inconexas que aportan nuevo conocimiento a viejos temas, que redefinen lo que parecía estable y definitivamente sabido, y que apoyan nuevos modos de pensar.

Así está surgiendo una conceptualización de la episteme popular (Scannone, 1990; Moreno, 1993) como modo de conocer el mundo paralelo, contigüo y, a la vez, entrecruzado con los modos estatuidos. Y a partir de ella una episteme de la relación, que reconoce la importancia y el carácter fundante de la afectividad y que supone a su vez una ontología de la relación, es decir, un sujeto plural formado, como ya lo habían anunciado Buber (1969) y Levinas (1972), en conceptos primordiales en los cuales el yo no es separable del $t u$. Un sistema de comprensión del mundo en función de las relaciones en las cuales somos, pues nadie puede ser con prescindencia del Otro. Esta es la vía para comprender el aspecto ético del paradigma de la relación: sólo cuando aceptamos al Otro como parte de nosotros nos liberamos y liberamos al Otro. Oprimir al Otro, someterlo, nos priva de nuestra propia humanidad.

Y, asimismo, al lado de la dialéctica, Dussel (1973) nos propone un nuevo método que igualmenle acepte a ese otro fundamental para ser, y con el cual estamos y somos: la analéctica, un camino para reestructurar la dialéctica aceptando como polo antitético, no sólo a aquel que es mi otra cara, mi opuesto definido a partir de mi ser, sino a aquel que es distinto, tanto que no puedo preverlo. El que viene de afuera, de un lugar otro. Se propone así una anadia-léctica que expande el alcance de la construcción del conocimiento. Lo interesante y lo liberador es que este movimiento latinoamericano parece estar arribando, por muchos caminos, a un conocimiento del Otro, no a partir del yo o del nosotros, sino a partir de ese Otro mismo que mientras más distinto $y$, por lo tanto, menos yo, ha sido tantas veces excluido, tantas veces oprimido. La historia de América Latina ilustra bien las exclusiones y las opresiones, pero también es una historia de liberaciones y de formas de acción solidarias. Y en ellas debe apoyarse esta forma de hacer psicología. De tal manera que, al final, como al principio, liberar sea un verbo que se conjugue en plural. 


\section{Referencias bibliográficas}

Blanco, A., (coord.), Ignacio Martín-Baró. Psicología de la Liberación, Madrid, 1998.

Blanco, A., El desde dónde y el desde quién. Una aproximación a la obra de Ignacio, 1993.

Boff, C., Teología e prática, Petrópolis: Vozes, $3^{4}$ edición, 1993.

Buber, M., Je et $\mathrm{tu}$, Paris: Aubier, 1969.

Dussel, E., Para una ética de la liberación latinoamericana, Buenos Aires: Siglo XXI, 1973.

Fals Borda, O., Acción comunal en una vereda colombiana, Bogotá: Universidad Nacional, Monografías Sociológicas, 1959.

Fals Borda, O., Ciencia propia y colonialismo intelectual, Bogotá: Carlos Valencia, editor, 1981, pp. 209-271.

Fals Borda, O., Conocimiento y poder popular, Bogotá: Siglo XXI, 1985.

Fals Borda, O., "Por la praxis. Cómo investigar la realidad para transformarla", Ciencia y política en ciencias sociales. Simposio de Cartagena, Bogotá, Punta de Lanza, 1978.

Freire, P., La educación como prácrica de libertad, México: Siglo XXI, 1964.

Freire, P., Pedagogía del oprimido, México: Siglo XXI, 1990.

Gramsci, A., Textos de los cuadernos posteriores a 1931, en M. Sacristán (comp.), Antologia, México: Siglo XXI, 7 Edición, 1931/1984.

Guba, E., Dialog of paradigms, Newbury Park: Sage, 1991.

Levinas, E., Humanismo del otro hombre, México: Siglo XXI, 1972/1993.

Lincoln, Y. y Guba, E., Naturalistic Inquiry, Beverly Hills: Sage, 1985.

Martín-Baró, I., Comportamiento, Universidad "Simón Bolívar", Caracas, Venezuela, Vol. 2, 2, pp. 35-60.

Martín-Baró, l., "El latino indolente. Carácter ideológico del fatalismo latinoamericano". En M. Montero (coord.), Psicología política latinoamericana, Caracas: Panapo, 1987, pp. 135-162.
Martín-Baró, I., "Hacia una psicología de la liberación", Boletín de Psicología, Universidad Centroamericana "José Simeón Cañas", San Salvador, 1986.

Martín-Baró, I., "Los grupos con historia: un modelo psicosocial", Boletín de la AVEPSO, XI (1), 1988, pp. 3-18.

Martín-Bar6, I., "Retos y perspectivas de la Psicología en América Latina", en G. Pacheco y B. Jiménez (comps.), Ignacio Martín-Baró (1942-1989) Psicología de la Liberación para América Latina, Guadalajara: Universidad de Guadalajara-ITESO, 1989/ 1991.

Montero, M., "Concientización, conversión y desideologización en el trabajo psicosocial comunitario", Boletín de la AVEPSO, XIV (1), 1991, pp. 3-12.

Montero, M., "Psicología de la liberación. Propuesta para una teoría psicosociológica", en H. Riquelme (comp.), Otras realidades, atras vias de acceso, Caracas: Nueva sociedad, 1991, pp. 133-150.

Moreno, A., El aro y la trama, Caracas: CIP.

Prilleltensky, I. y Gonick, L., The discourse of opression: towards an emancipatory social science praxis, 1993.

Prilleltensky, I., The morals and politics of Psychology, New York: University of New York Press, 1994.

Scannone, J.C., Nuevo punto de partida de la filosofia latinoamericana, Buenos Aires: Guadalupe, 1990.

Serrano-García, I. y López, G., "Una perspectiva diferente del poder y el cambio social para la psicología social comunitaria", en M. Montero (coord.), Psicología social comunitaria. Teoría, método y experiencia, Caracas: EDUVEN, 1991, pp. 167-210.

Theo, T., "Prolegomenon to a contemporary psychology of liberation", Theory and Psychology, 8 (4), 1998, pp. 527-548.

Williamsburg, VA, EE.UU., Ponencia en la IV Conferencia bienal de la Society for community research and action, División 27, A.P.A

Rodríguez, A., Introducción a la Psicología Social, Petrópolis, Vozes, 1972. 